\title{
Suffizienzorientiertes Onlinemarketing
}

\author{
Drei Erden verbraucht Deutschland nach Berechnungen \\ des Global Footprint Network pro Jahr - wir haben die Grenzen \\ nachhaltigen Lebens und Wirtschaftens längst überschritten. \\ Kompensation, ökologischer und fairer Konsum sind vor diesem \\ Hintergrund immer nur die zweitbeste Lösung. \\ Von Stephanie Keilholz
}

\section{D} as derzeitige Wirtschaftsmodell folgt einem Leitbild: steigende Profite durch Wachstum und Kostensenkungen. Innerhalb dieses Paradigmas haben in den vergangenen Jahren zwei Strategien für mehr Nachhaltigkeit einen Weg ins Marketing gefunden. Die Effizienzstrategie mit den Kernbegriffen „besser“, „effizient" und „sparsam“ zielt auf eine erhöhte Ressourcenproduktivität bei gleichbleibender Abverkaufsmenge ab.

Der Einsatz von naturverträglichen Technologien und Ressourcen bei gleichbleibender Abverkaufsmenge steht im Mittelpunkt der Konsistenzstrategie (von Winterfeld 2007; Griese/Halstrup 2013). Die dritte und vor dem Hintergrund akuter gesellschaftlicher Herausforderungen wichtigste Strategie, die Suffizienzstrategie, findet in der unternehmerischen Praxis kaum Anwendung (Griese/ Halstrup 2013).

\section{Bedarf und Herausforderungen}

Die Umsetzung stellt Unternehmer/ innen vor Herausforderungen, da die für Suffizienz erforderliche Reduktion von Volumen und Frequenz einen Widerspruch zur gängigen Wachstumspraxis darstellt. Technischer Fortschritt hin $\mathrm{zu}$ effizienterem oder anderem Ressourceneinsatz wird deshalb oftmals als entscheidende Lösung für Nachhaltigkeit angesehen.
Im Onlinemarketing verfolgen konsistenz- oder effizienzorientierte Strategien die Zielsetzung, ein nachhaltigeres Produkt an möglichst viele Menschen zu vermarkten. Wenn effizientere Produkte dadurch intensiver genutzt werden oder zu weiteren Neuanschaffungen führen, werden technische Ressourceneinsparungen durch Rebound-Effekte zunichte gemacht (Seidl/Zahrnt 2016). Um soziale und ökologische Konsequenzen von Produktion und Konsum angemessen zu berücksichtigen, sollten Konsistenz, Effizienz und Suffizienz in der Marketing- und Unternehmensstrategie deshalb simultan eingesetzt werden.

\section{Suffizienzorientierung im Onlinemarketing}

In der suffizienzorientierten Kommunikation wird der Fokus auf einen konsumentenseitig genügsamen und umweltverträglichen Verbrauch von Material- sowie Energiemengen bei gleichzeitig steigendem individuellen Nutzengewinn beziehungsweise einer steigenden Lebensqualität gelegt (Griese/ Halstrup 2013).

Die Intensität und Ausrichtung von Onlinemarketing-Maßnahmen werden durch ein kontinuierliches Abwägen der Notwendigkeit begrenzt. Es geht im Kern darum, so viel zu vermarkten, wie nötig und so wenig wie möglich. Für die strategische Zielsetzung der Marketingaktivitäten wird der gesamte Konsumpro- zess vom tatsächlichen Bedürfnis über die Herstellung bis zur Entsorgung, Reparatur oder Weitergabe betrachtet. Mittels Onlinemarketing kann Suffizienz attraktiv dargestellt, können suffiziente Konsumweisen gefördert und überlegte Entscheidungen ermöglicht werden (Bewusstseinsbildung). In der Kommunikation und Werbung liegt der Fokus weniger auf Produktfeatures und Preisen, sondern vor allem auf Wertschätzung, Genuss und Verantwortung. Informative und inspirierende Inhalte treten an die Stelle von reiner Produktwerbung. Suffizienzorientierte Botschaften wie „Buy Less, But Better" (Patagonia-Kampagne 2011) oder auch Hinweise auf Kaufalternativen (Leihen, Tauschen, Reparieren, Selbermachen) können eine zentrale Rolle spielen.

Unternehmer/innen können Technologie nutzen, um suffizienten Konsum zu ermöglichen. Ein Onlineshop kann zum Beispiel sparsamere Produktalternativen prominenter platzieren oder einen Ressourcenverbrauchsfilter für die Recherche einbauen.

\section{Potenziale}

Eine empirische Untersuchung $(\mathrm{n}=1.616)$, die 2018 von einer Forschungsgruppe des IÖW und der Technischen Universität Berlin in Zusammenarbeit mit dem nachhaltigen Onlineshop Avocado Store durchgeführt wurde, gibt Anhaltspunkte im Hinblick auf die Wirksamkeit und Akzeptanz suffizienzorientierter Marketingansätze.

In der Untersuchung wurde den Besucher/innen während des Einkaufsvorgangs ein Entscheidungsbaum als Pop-up eingeblendet. Dieser startete mit der Leitfrage „Brauchst du das wirklich?", um in verschiedenen Iterationen eine bewusste Kaufentscheidung der Konsument/innen zu fördern. Die Befragten reagierten überwiegend positiv auf diese suffizienzorientierte Onlinemarketing-Maßnahme, der Absender wurde vertrauenswürdig, glaubhaft und qualifiziert wahrgenommen, die inhaltliche Botschaft sprach die Menschen zumeist an. 
Suffizienzorientiertes Onlinemarketing bietet Potenziale für eine differenzierte Positionierung im eigenen Marktsegment. Außerdem können Unternehmen mit suffizienzorientierter Kommunikation einen Beitrag zur Entstehung und Verbreitung suffizienter Lebensweisen leisten (Griese/Halstrup 2013). Der Einstieg in suffizienzorientiertes Onlinemarketing ist niedrigschwellig möglich, da digitale Maßnahmen kurzfristig umgesetzt werden können, während für die Umstellung von Produktionsprozessen oder des gesamten Geschäftsmodells tiefergehende Strukturveränderungen notwendig sind.

\section{Dialog über Onlinekanäle}

Onlinekanäle wie Social Media, E-Mail-Marketing und Blogs ermöglichen es, die Reise zu mehr Nachhaltigkeit in unternehmerischen Prozessen transparent zu machen und Konsument/ innen in Form eines stetigen Dialogs direkt in aktuelle Entwicklungen einzubinden. Zukunftsweisende Unternehmen achten bereits in der Geschäfts- und Produktentwicklung darauf, dass Suffizienz und damit suffizienzorientiertes Marketing möglich wird.

Dies kann neben der ökologischen und fairen Produktion zum Beispiel durch die Langlebigkeit der Produkte, zeitloses Design oder Reparaturmöglichkeiten realisiert werden. Auch Sharing-, Tausch- oder Leihmodelle als Ergänzung oder Hauptbestandteil des Geschäftsmodells können gut geeignet sein, um Konsumentenbedürfnisse zu befriedigen und gleichzeitig soziale und ökologische Konsequenzen des Wirtschaftens zu berücksichtigen.

\section{Teil der Nachhaltigkeits- strategie}

Nicht zuletzt trägt suffizienzorientiertes Onlinemarketing als Teil einer übergreifenden Nachhaltigkeitsstrategie zur Sicherung der Lebensgrundlagen und zum Erhalt von Produktivität bei (von Winterfeld 2007), da soziale und ökologische Faktoren zumindest gleichwertig mit Gewinnbestrebungen in das unternehmerische Handeln einfließen. Suffizienzorientiertes Onlinemarketing benötigt einen ganzheitlichen Ansatz, um zu funktionieren - vom Geschäftsmodell über das Produktdesign bis hin zur Onlinekommunikation.

\section{Fazit}

Es gibt genügend Gründe für suffizienzorientiertes Onlinemarketing. Nun braucht es mutige Unternehmen und Vermarkter/innen, die eine Balance zwischen ausreichendem Umsatz und Ressourcenschonung finden und so $\mathrm{zu}$ maßvollem Konsum bei steigender Lebensqualität für alle beitragen.

\section{Literatur}

Global Footprint Network (2019): Country Overshoot Days. Im Internet unter: https://www.overshootday.org/newsroom/ country-overshoot-days.

Griese, K.-M./Halstrup, D. (2013): Umsetzung einer suffizienzorientierten Unternehmenskommunikation und die Bedeutung der Stakeholder: Ansätze und Empfehlungen. In: uwf UmweltWirtschaftsForum ISSN 0943-3481, Volume 21, Combined 1-2.

Griese, K.-M./Halstrup, D. (2013): Suffizienz in der Unternehmenskommunikation. In: Ökologisches Wirtschaften, 4.2013, S. 40-43.

Seidl, I./Zahrnt, A.: Effizienz oder Suffizienz? Effizienz und Suffizienz! In: movum, Ausgabe 10, Februar/2016, S. 6.

Von Winterfeld, U. (2007): Keine Nachhaltigkeit ohne Suffizienz. Fünf Thesen und Folgerungen. In: vorgänge 179, Zeitschrift für Bürgerrechte und Gesellschaftspolitik, Heft 3, September 2007, Berliner WissenschaftsVerlag, S. 46-54.

AUTORIN + KONTAKT

Stephanie Keilholz ist Diplom-Betriebswirtin und Gründerin der öko-sozialen Kreativagentur „Das Gute Ruft“.

Das Gute Ruft, Adolphstraße 18, 50679 Köln. Tel.: +49 221 7900-4233, E-Mail: hallo@dasguteruft.de

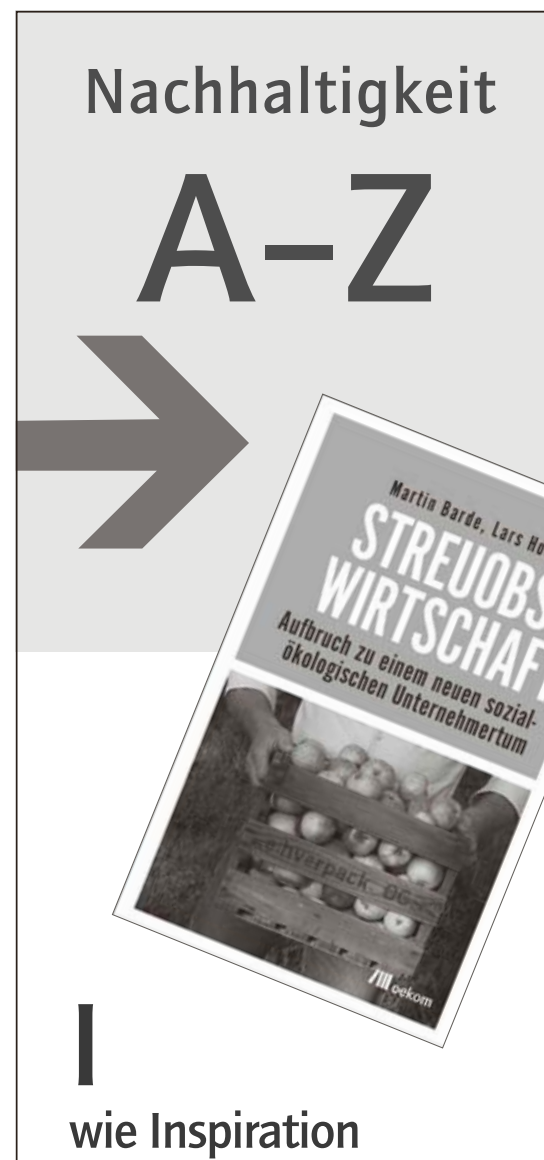

In der Land- und Ernährungswirtschaft findet heute viel zu oft eine effizienzgetriebene Übernutzung von Natur und Ressourcen statt - auf Kosten von Vielfalt und Lebendigkeit. Martin Barde und Lars Hochmann zeigen anhand des Beispiels Streuobstwirtschaft, wie es besser geht: mit einem neuen sozialökologischen Unternehmertum und mit Menschen, die Missstände nicht länger hinnehmen, sondern etwas unternehmen, damit es besser wird!

M. Barde, L. Hochmann Streuobstwirtschaft Aufbruch zu einem neuen sozialökologischen Unternehmertum

192 Seiten, Hardcover, 24,- Euro, ISBN 978-3-96238-092-2

Erhältlich im Buchhandel oder versandkostenfrei innerhalb Deutschlands bestellbar unter www oekom de Auch als E-Book erhältlich.

\section{/II oekom}

Die guten Seiten der Zukunft 